\title{
Novae and Accreting White Dwarfs as Progenitors of Type Ia Supernovae
}

\author{
Mariko Kato \\ Keio University, \\ 4-1-1, Hiyoshi, Kouhoku-ku Yokohama, 223-8521 Japan \\ email: mariko@educ.cc.keio.ac.jp
}

\begin{abstract}
I review various phenomena associated with mass-accreting white dwarfs (WDs) in relation to progenitors of Type Ia supernovae (SNe Ia). The WD mass can be estimated from light curve analysis in multiwavelength bands based on the theory of optically thick winds. In the single degenerate scenario of SNe Ia, two main channels are known, i.e., WD + main sequence (MS) channel and WD + red giant (RG) channel. In each channel, a typical binary undergoes three evolutionary stages before explosion, i.e., the wind phase, supersoft X-ray source (SSS) phase, and recurrent nova phase, in this order because the accretion rate decreases with time as the companion mass decreases. For some accreting WDs we can identify the corresponding stage of evolution. Intermittent supersoft X-ray sources like RX J0513.9-6951 and V Sge correspond to wind phase objects. For the SSS phase, CAL 87-type objects correspond to the WD+MS channel. For the WD + RG channel, soft X-ray observations of early type galaxies give statistical evidence of SSS phase binaries. Recurrent novae of U Sco-type and RS Oph-type correspond to the WD + MS channel and WD + RG channel, respectively. The majority of recurrent novae host a very massive WD $\left(\gtrsim 1.35 M_{\odot}\right)$ and often show a plateau phase in their optical light curves corresponding to the long-lasting supersoft X-ray phase. These properties are indications of increasing WD masses.
\end{abstract}

Keywords. stars: novae, supernovae - X-rays: stars, binaries

\section{Introduction}

Mass accreting white dwarfs (WDs) become novae, persistent supersoft X-ray sources (SSSs), or wind evolution objects, depending on the mass-accretion rate. Very massive WDs and their position in binary evolution are interesting subjects because they are closely related to Type Ia supernovae (SNe Ia) progenitors. In this paper I review how to specify the mass and its growth rate for WDs, and discuss them in relation to progenitors of SNe Ia. Section 2 introduces binary evolution scenarios to SNe Ia in which accreting WDs are growing in mass (single degenerate scenario). For relatively low mass accretion rates $\left(\lesssim 1 \times 10^{-7} M_{\odot} \mathrm{yr}^{-1}\right)$, the WDs experience recurrent nova outbursts. Section 3 describes how to determine the WD mass from the light curves of novae and specifies mass-increasing WDs. With intermediate accretion rates, hydrogen burning is stable and WDs become persistent supersoft X-ray sources (SSSs), which are the subject of Section 4. For high mass-accretion rates, optically thick winds inevitably occur. Such objects are described in Section 5. Section 6 introduces helium novae and shows the mass accumulation efficiency during helium shell-flashes.

\section{Binary Evolutions Toward Massive WDs}

Figure 1 shows the map of response of WDs to mass-accretion rates. When the massaccretion rate onto the WD is smaller than $\dot{M}_{\text {st }}$, hydrogen burning is unstable and nova 


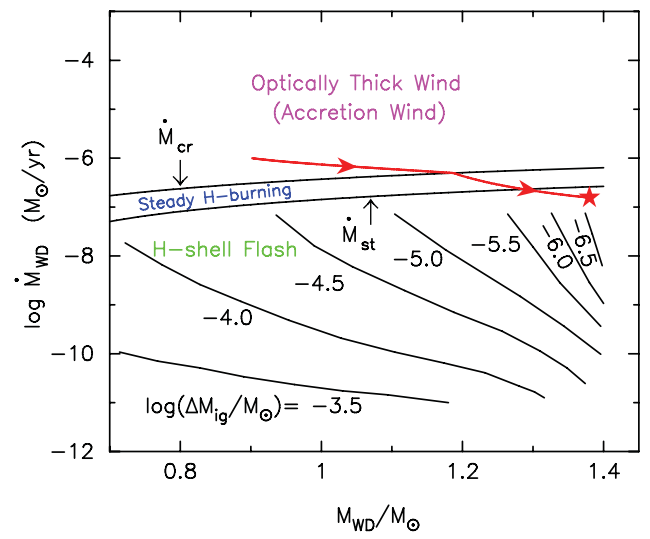

Figure 1. A typical evolutionary path (red solid line) of an SN Ia progenitor (in the SD scenario) on the map of response of WDs to mass accretion rate. Starting from the accretion wind phase, in which strong optically thick winds blow from the WD, the binary enters the supersoft X-ray phase, which occupies a narrow region of accretion rates between $\dot{M}_{\mathrm{cr}}>\dot{M}_{\mathrm{WD}}>\dot{M}_{\mathrm{st}}$. When the mass accretion rate decreases, to less than the value to keep steady hydrogen-burning $\left(\dot{M}_{\mathrm{st}}\right)$, the binary enters the recurrent nova (weak shell flashes) phase. The WD explodes at the star mark as an SN Ia (taken from Hachisu et al. 2010).
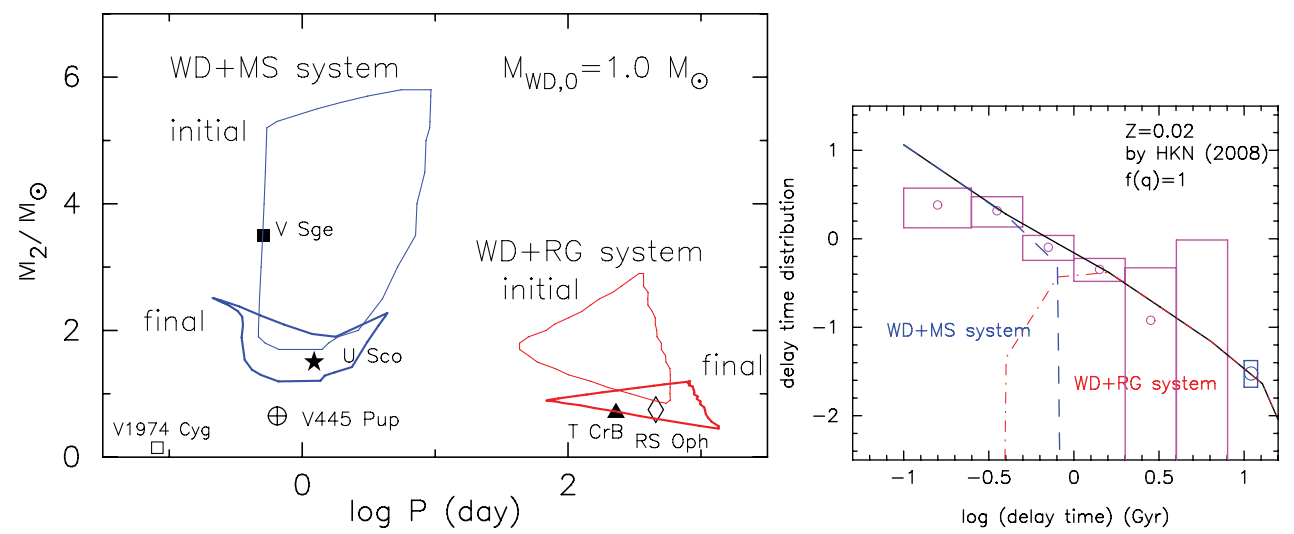

Figure 2. Left: The regions that produce SNe Ia are plotted in the orbital period - secondary mass plane for the (WD + MS) system (left) and (WD + RG) system (right). Here we assume a metallicity of $Z=0.02$ and the initial WD mass of $M_{\mathrm{WD}, 0}=1.0 M_{\odot}$. The initial system inside the region encircled by a thin solid line (labeled "initial") increases its WD mass up to the critical mass $\left(M_{\text {Ia }}=1.38 M_{\odot}\right)$ for the SN Ia explosion, the regions of which are encircled by a thick solid line (labeled "final"). Currently known positions of individual objects are plotted for recurrent novae: U Sco (Hacisu et al. 2000), T CrB (Belczynski \& Mikołajewska 1998), RS Oph (Brandi et al. 2009), a supersoft X-ray source: V Sge (Hachisu et al. 2003), classical novae: V1974 Cyg (Hachisu \& Kato 2006), and the helium nova: V445 Pup (Goranskij et al. 2010, Kato et al. 2008). Right: Delay time distribution based on the SD scenario. Contributions of the two channels reproduce well the observational results (taken from Hachisu et al. 2008).

outbursts (classical nova: $\mathrm{CN}$ ) are triggered periodically. If the mass-accretion rate is relatively large and close to the upper limit, $\dot{M}_{\text {st }}$, the WD experiences weak shell flashes with a short recurrence period. Such objects are recurrent novae (RNe). In the intermediate mass-accretion rate of $\dot{M}_{\mathrm{st}}<\dot{M}_{\mathrm{acc}}<\dot{M}_{\mathrm{cr}}$, hydrogen shell-burning is stable and the mass-accreting WD becomes a persistent SSS in which the mass-accretion rate is equal to the mass consuming rate owing to nuclear burning. For a higher mass accretion rate 
$\left(\dot{M}_{\mathrm{cr}}<\dot{M}_{\text {acc }}\right)$ hydrogen burning cannot consume all of the accreted material, the rest of which expands, and an optically thick wind blows. In the traditional double degenerate (DD) scenario (e.g. Iben \& Tutukov 1984), such a binary is considered to undergo a common envelope evolution that leads finally to a pair of degenerate stars. In the beginning of 1990, however, opacity tables were revised. The new opacities (e.g., OPAL opacities) show a strong peak at $\log T(\mathrm{~K}) \sim 5.2$, which brings significant structure changes to WD envelopes; i.e., the optically thick winds are accelerated and the envelope photosphere does not reach the companion's orbit so the binary does not experience a common envelope evolution in $\dot{M}_{\mathrm{cr}}<\dot{M}_{\text {acc }} \lesssim 10^{-4} M_{\odot} \mathrm{yr}^{-1}$. Therefore, the binary evolution was essentially changed in a large part of the upper region of Figure 1 (Hachisu et al. 1996).

These optically thick winds were incorporated into binary evolution scenarios and two paths in which the WD grows in mass toward the Chandrasekhar mass were found. One is the WD + main sequence (MS) channel (Li \& van den Heuvel 1997; Hachisu et al. 1999a) and the other is the WD + red giant (RG) channel (Hachisu et al. 1999b).

Figure 1 shows a typical evolutionary path to SN Ia explosion, which is common to both the channels. A binary starts the accretion wind phase, because the mass transfer rate onto the WD is larger than the critical rate for the occurrence of optically thick winds $\left(\dot{M}_{\mathrm{cr}}\right)$. So the binary undergoes accretion wind evolution (see Section 5 for more details). As the mass transfer rate decreases with time, the wind stops and the binary enters the supersoft X-ray phase. Steady hydrogen burning occurs in both the wind and SSS phases, and the WD mass continuously increases. When the mass accretion rate further decreases to less than the value needed to keep steady hydrogen-burning $\left(\dot{M}_{\mathrm{st}}\right)$, the binary enters the recurrent nova phase. The shell flashes are weak and a part of the accreted matter remains after each outburst, so the WD mass continuously increases. The WD explodes as an SN Ia at the star mark in Figure 1.

Figure 2 (left) shows the initial/final regions of the binary evolutions in which the WD grows its mass to the critical mass $\left(M_{\text {Ia }}=1.38 M_{\odot}\right)$ and explodes as an SN Ia. The binary inside the regions (labeled "initial") evolves down to inside the "final" region and explodes there.

This figure also shows three RNe, a wind phase object (V Sge), a classical nova (V1974 Cyg), and a helium nova (V445 Pup) with the known orbital period and suggested secondary mass. The position of three RNe (U Sco, RS Oph and T CrB) are very consistent with the final region, and V Sge (still midway of evolutionary path downward) is also consistent. The classical nova V1974 Cyg is located below the final region of the WD+MS system, which indicates that they are not SNe Ia progenitors (see Section 3).

These two different paths to SNe Ia explain well the observed delay time distribution as shown in Figure 1 (right). The WD+MS channel corresponds to the prompt component and WD+RG channel the tardy component (see Hachisu et al. 2008 for details).

\section{Recurrent Novae}

Nova light curves are calculated using the optically thick wind theory of nova outbursts for a given set of the WD mass and chemical composition of the envelope (Kato \& Hachisu 1994). The optical and infrared (IR) fluxes are basically well represented by free-free emission. The supersoft X-ray light curve is calculated from blackbody emission, which may be inaccurate but is sufficient for estimating the X-ray turn on/off time.

Figure 3 shows the observed multi-wavelength light curves of RS Oph and V2491 Cyg. After the optical maximum, the photospheric temperature rises with time while the total luminosity is almost constant. Therefore, the main emission region shifts from optical to $\mathrm{UV}$ and then to supersoft X-rays. The decline rate of the optical flux and the duration 
days after maximum
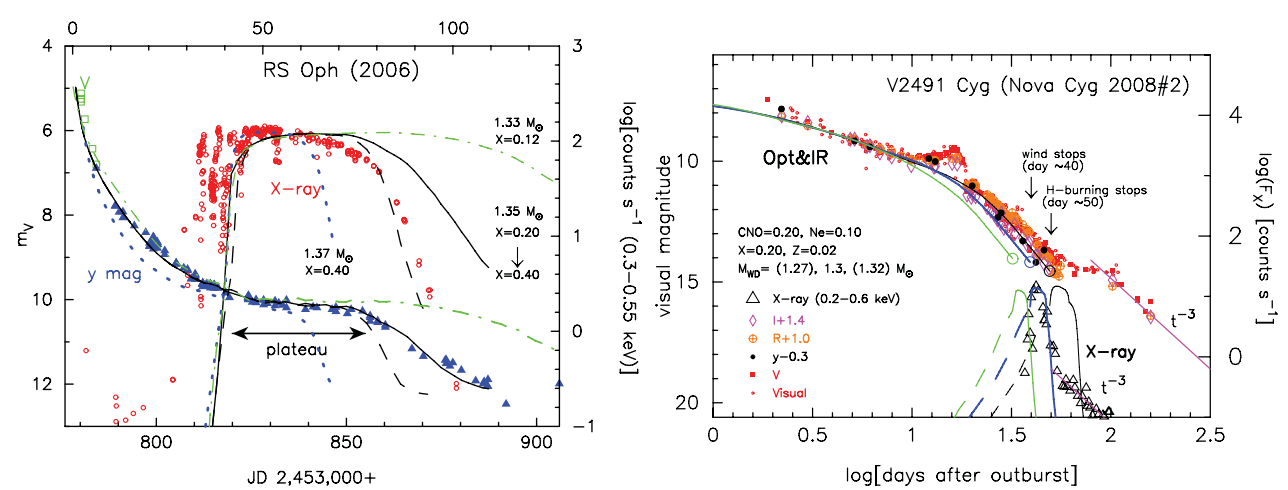

Figure 3. Left: Light curve fitting of the recurrent nova RS Oph. Observed light curve: Optical magnitude (green open squares and blue filled triangles), and supersoft X-ray count rates (red open circles). Lines indicate model light curves: $M_{\mathrm{WD}}=1.33 M_{\odot}$ (dash-dotted line), $1.35 M_{\odot}$ (solid line), and $1.37 M_{\odot}$ (dashed line). This figure is taken from Hachisu et al. (2007). Right: Light-curve fitting for the classical nova V2491 Cyg. The upper bunch of data points indicates optical and near-IR observational data, and the lower black triangles represent X-ray data. The best-fit theoretical model (thick blue line) is a $1.3 \mathrm{M}_{\odot} \mathrm{WD}$ with an envelope chemical composition of $X=0.20, Y=0.48, X_{\mathrm{CNO}}=0.20, X_{\mathrm{Ne}}=0.10$, and $Z=0.02$. Supersoft X-rays may not be detected during the wind phase (dashed part) because of self-absorption by the wind itself. The $F_{\lambda} \propto t^{-3}$ law is added for the nebular phase. See Hachisu \& Kato (2009a) for more details.

of the X-ray phase depend differently on the WD mass and composition. This property is useful in determining the WD mass.

Figure 3 (left) also shows the light-curve fitting of the recurrent nova RS Oph. The light curve model consists of a $1.35 M_{\odot}$ WD, a $0.7 M_{\odot}$ RG companion with a radius of $35 R_{\odot}$ and an irradiated disk with a radius of $47 R_{\odot}$ around the white dwarf. The binary orbital period is 455.72 days and the inclination angle is $i=33^{\circ}$. The model produces reasonable agreement with the observation of both the supersoft X-ray and optical observations (see Hachisu et al. 2006 for more details).

The light-curve fitting of V2491 Cyg is shown in Figure 3 (right). V2491 Cyg is a very fast $\mathrm{CN}$ that shows an early decline very similar to that of $\mathrm{RS}$ Oph (it may not look it because the left figure is plotted on a linear scale while the right one is on the logarithmic scale), but shows a very short supersoft X-ray phase of only 10 days. The best fit model, a $1.3 M_{\odot}$ WD, reproduces simultaneously the light curves of visual, IR, and X-ray, except the secondary maximum about 15 days after the optical peak, which was explained as having a magnetic origin (see Hachisu and Kato 2009a). In the very late phase, the visual light curve deviates from the theoretical lines due to contribution of strong emission lines.

In general, a nova evolves faster in a more massive WD and slower in a less massive WD. Therefore, a very fast decline of the optical light curve is an indication of a very massive WD, but not all of the massive WDs are candidate SNe Ia progenitors. For example, V2491 Cyg shows a fast optical decline in the early phase very similar to RS Oph, and the WD mass is estimated to be as massive as $\sim 1.35 M_{\odot}$ in RS Oph and $\sim 1.3 M_{\odot}$ in V2491 Cyg. RS Oph shows no indication of heavy element enhancement in its ejecta, which is consistent with a mass-increasing WD. Implementing detailed light curve analysis of RS Oph, Hachisu et al. (2007) derived the mass-growth rate of the WD to be $(0.6-1) \times 10^{-7} M_{\odot} \mathrm{yr}^{-1}$. On the other hand, V2491 Cyg is a classical nova, and 
heavy element enhancement of ejecta is observed (Munari et al. 2011). This indicates that the WD mass is decreasing, because the surface layer of the WD was eroded during the outburst.

It is interesting to compare the durations of the SSS phase in RS Oph and V2491 Cyg. The SSS phase of RS Oph lasts as long as 60 days, whereas only 10 days in V2491 Cyg. The long lasting SSS phase in RS Oph is an indication of a hot helium ash layer produced by hydrogen burning. This hot helium ash acts as a heat reservoir to keep the WD temperature high enough to emit supersoft X-rays for a long time (Hachisu et al. 2007). RS Oph shows a plateau phase in the optical light curve which is explained as a contribution of the irradiated disk heated by the hot WD (Hachisu et al. 2006). The sharp end of the plateau phase corresponds to the turnoff of supersoft X-rays (Hachisu et al. 2007). V2491 Cyg shows a very short duration, which indicates the absence of a helium ash layer, consistent with a mass-decreasing WD.

The classical nova V838 Her also shows a very similar optical light curve to that of U Sco. V838 Her is a classical nova with very massive WD $\left(\sim 1.35 M_{\odot}\right)$ and heavy element enrichment is observed in its ejecta (see Table 2 in Kato et al. 2009) This indicates that the WD mass is decreasing. On the other hand, U Sco is a recurrent nova in which the WD mass is increasing at an average rate of $\sim 1 \times 10^{-7} M_{\odot} \mathrm{yr}^{-1}$ (Hachisu et al. 2000). U Sco also shows a plateau phase of 18 days corresponding to the SSS phase. This is also indicative of a helium heat reservoir, i.e., the WD mass is increasing.

It should be noted that classical nova binaries undergo different evolutionary paths from recurrent novae, and do not produce SNe Ia. Figure 2 (left) shows that some classical novae are outside of the initial/final region of promising binary regions to $\mathrm{SNe}$ Ia, whereas $\mathrm{RNe}$ are located within the final region because their WD mass is close to the final value of $1.38 M_{\odot}$.

\section{Supersoft X-ray Sources}

At intermediate mass-accretion rates $\left(\dot{M}_{\mathrm{st}} \leqslant \dot{M}_{\mathrm{WD}} \leqslant \dot{M}_{\mathrm{cr}}\right)$, hydrogen burning is stable and optically thick winds do not occur. van den Heuvel et al. (1992) interpreted supersoft X-ray sources like CAL 83 and CAL 87 in the Large Magellanic Cloud (LMC) as accreting WDs with high mass-accretion rate $\left(\approx 10^{-7} M_{\odot} \mathrm{yr}^{-1}\right)$ with steady hydrogen nuclear burning. The distribution of the LMC SSSs in an HR diagram is consistent with the theoretical SSS region of accreting WDs. The photospheric temperature of the WD is relatively high, $\log T(\mathrm{~K})=5.4-6.0$, so the WDs emit supersoft X-rays (Nomoto et al. 2007). Note that the LMC SSSs are all close binaries, which correspond to the SSS phase of the WD + MS channel. There are two SSS symbiotic stars in SMC (SMC3 and Lin358). They may correspond to the SSS phase of the WD + RG channel, although the WD mass is not well determined. Their surface temperatures are relatively $\operatorname{low}, \log T(\mathrm{~K})=5.5$ and 5.7 for Lin358 and SMC3, respectively, which may be one of the reasons for relatively low X-ray emission.

Recently, supersoft X-ray fluxes of early type galaxies were obtained by Gilfanov \& Bogdán (2010). In early type galaxies the star formation ended long time ago, so the progenitor binaries are all the WD+RG systems. The X-ray fluxes estimated by Gilfanov \& Bogdán (2010) are very consistent with model X-ray fluxes based on the SD scenario, which is a strong support to the SD scenario of SNe Ia, although Gilfanov \& Bogdán drew an opposite conclusion (see Kato 2011, Hachisu et al. 2010 for more details). 


\section{Accretion Wind Phase}

When the accretion rate is larger than $\dot{M}_{\text {cr }}$, the WD cannot consume all of the accreted matter by nuclear burning, so the accreted matter is piled up to form an extended envelope. As the photospheric temperature decreases to below the critical value, optically thick winds are accelerated due to Fe peak (at around $\log T(\mathrm{~K}) \approx 5.2$ ) of the OPAL opacity (Kato and Hachisu 1994, 2009).

Hachisu and Kato (2001) proposed a binary system in which the WD accretes matter from the companion from the equatorial region and loses matter as a wind from the other regions as illustrated in Figure 4 (left). They called such a configuration "accretion wind." In this situation, the WD burns hydrogen at the rate of steady burning $\dot{M}_{\text {nuc }}$ and blows the rest of the accreted matter in the winds at the rate of about $\dot{M}_{\mathrm{WD}}-\dot{M}_{\text {nuc }}$. WDs in this "accretion wind" correspond to the upper part of Figure 1.

Two SSSs, RX J0513.9-6951 and V Sge, correspond to this accretion wind evolution. RX J0513.9-6951 is an LMC supersoft X-ray source that shows quasi-regular transition between optical high and low states as shown in Figure 4 (right). Supersoft X-rays are detected only in the optical low states (Reinsch et al. 2000; Schaeidt, Hasinger, and Truemper 1993).

Hachisu and Kato (2003b) presented a transition mechanism between the high and low states. In the optical high state, the accretion rate is high enough and the photosphere expands to accelerate winds. The WD has a low surface temperature and no X-rays are expected. In the optical low state, the mass-accretion rate is low and the photospheric temperature is high enough to emit supersoft X-rays. No wind is accelerated. The authors proposed a self-regulation transition mechanism that makes the binary back and forth between the optical high and low states. When the mass-accretion rate is large, the WD is in the optical high state. The strong winds hit the companion and strip off a part of the companion surface. Thus the mass-transfer rate onto the WD reduces and finally stops, which causes the wind to stop and the system goes into the optical low state. After a certain time, the companion recovers to fill the Roche lobe again and the mass transfer resumes, which causes wind mass loss. The resultant theoretical light curves depend on
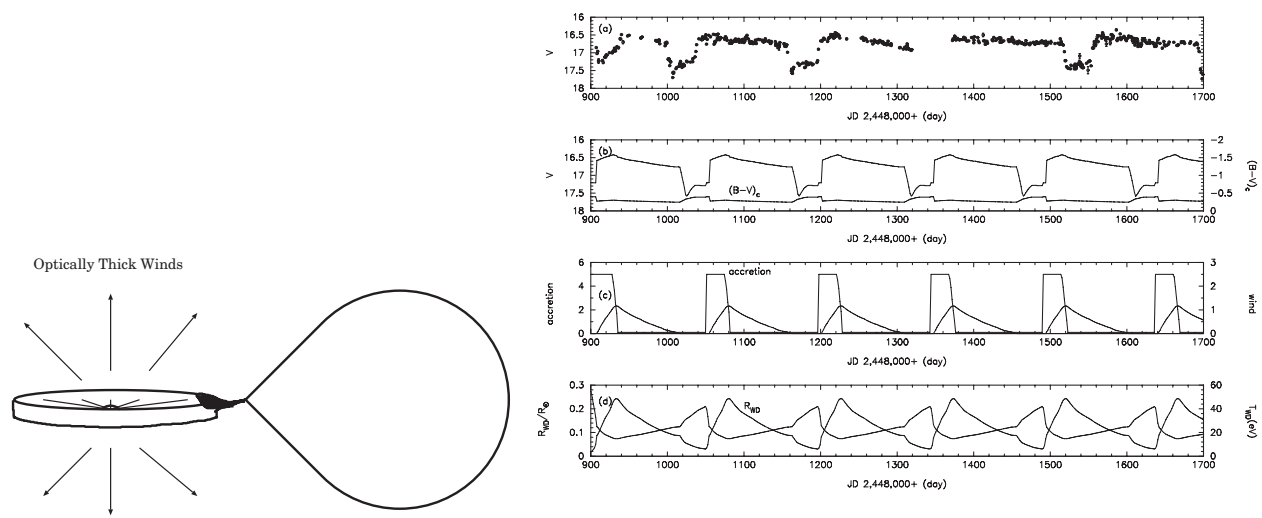

Figure 4. Left: Optically thick winds blow from mass-accreting WDs when the mass-transfer rate from a lobe-filling companion exceeds a critical rate, i.e., $\dot{M}_{\text {acc }}>\dot{M}_{\text {cr }}$. The white dwarf accretes mass from the equatorial region and at the same time blows winds from the polar regions. Stable hydrogen nuclear burning occurs on the surface of the WD. Right: Self-sustained model of spontaneous winds for supersoft X-ray source RX J0513-6951. (a) Long term evolution of $\mathrm{V}$ magnitude. (b) Model light curve of $M_{\mathrm{WD}}=1.3 M_{\odot}$. (c) Change of accretion rate and wind mass-loss from the WD envelope. (d) Change of WD radius and its temperature. (Taken from Hachisu \& Kato 2003a.) 
the WD mass and other parameters. The best fit model that reproduces the observed light curve indicates the WD mass to be 1.2 - $1.3 \mathrm{M}_{\odot}$ (Hachisu \& Kato 2003a).

The second object is V Sge that also shows a similar semi-regular transition of its light curve, although the timescales are different. Its light curve is also reproduced by the transition model with the WD mass of $1.2-1.3 \mathrm{M}_{\odot}$ (Hachisu \& Kato 2003b).

In these two systems the WD mass is increasing with time, because steady nuclear burning produces helium ash which accumulates on the WD. Therefore, they are candidates of SNe Ia progenitors. Figure 2 shows the position of V Sge in the initial/final binary diagram. The WD, of mass $1.2-1.3 \mathrm{M}_{\odot}$, is still increasing and the position of $\mathrm{V}$ Sge is on the way downward toward the final region.

These two objects, RX J0513.9-6951 and V Sge, suggest that the accretion wind evolution and stripping effects of the companion surface that regulate the mass-transfer rate from the companion are important and are actually working in binary evolution. This accretion wind is an important elementary process for binary evolution scenarios for Type Ia supernovae, because it governs the growth rate of the WD mass (e.g., Hachisu et al. 1999a, Hachisu et al. 1999b, Han \& Podsiadlowski 2006), as well as the masstransfer rate from the companion which is regulated by the stripping of the companion surface by the wind (e.g., Hachisu, Kato \& Nomoto 2008).

\section{He Novae}

A helium nova is a similar phenomenon to an ordinary nova, but the nuclear fuel is helium. If the companion is a helium star, the WD accretes helium and, when the accreted helium mass reaches a critical value, unstable helium burning triggers a thermonuclear runaway event. If the companion is a normal star, and the hydrogen-accreting WD is growing in mass (like recurrent novae or SSS), a helium ash layer develops underneath the hydrogen burning zone, which also triggers a helium nova outburst. Such helium novae were theoretically predicted by Kato et al. (1989) long before V445 Pup was discovered in 2000 December; V445 Pup has been the only helium nova so far.

Figure 5 (left) shows the light curve of V445 Pup, that shows a slow decline in $V$ and $I$ bands before the blackout due to dust formation. We have only 210 days of data available for a light curve analysis. From the light curve fitting, the WD mass is estimated

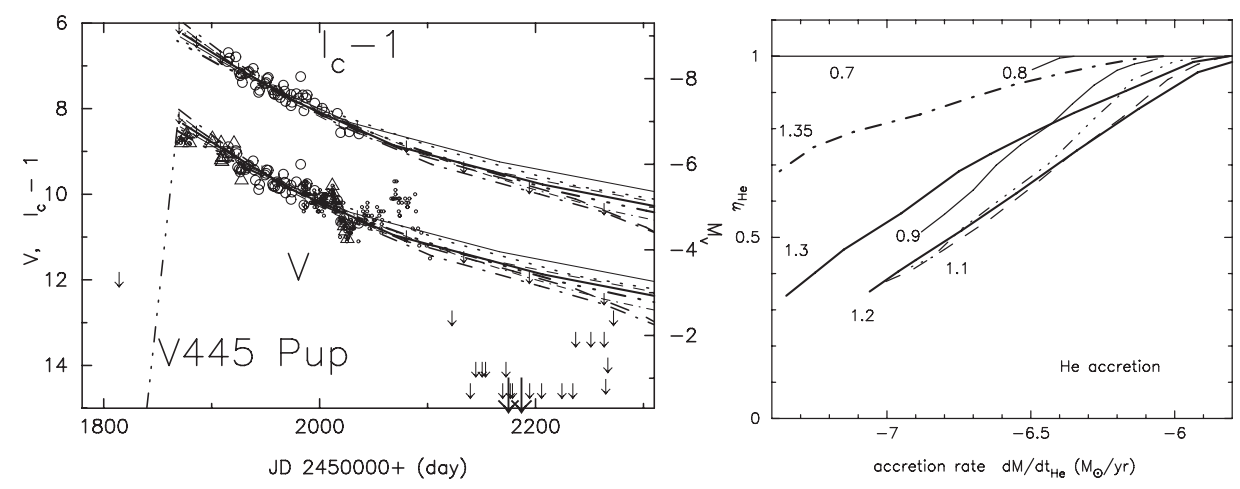

Figure 5. Left: Light curve fitting of the helium nova V445 Pup. Lines indicate the theoretical model for $1.35 M_{\odot}$ (dotted line), $1.377 M_{\odot}$ (dashed and dash-dotted lines), and $1.37 M_{\odot}$ (other lines) See Kato et al. (2008) for details. Right: Mass accumulation efficiency, $\eta_{\mathrm{He}}$, is plotted against the helium mass accretion rate. The WD mass is attached beside the curve. Figure taken from Kato \& Hachisu (2004). 
to be very high $\left(\geqslant 1.35 M_{\odot}\right)$ and the WD is growing in mass (see Kato et al. 2008 for more details). Therefore, V445 Pup is a candidate SN Ia progenitor. The companion is an evolved helium star but its evolutionary path is not well known; such a system of massive $\mathrm{WD}+$ helium star companion has no corresponding stage in the two known channels to SNe Ia (WD+MS channel and WD+RG channel).

Figure 5 (right) shows the mass accumulation efficiency $\eta_{\mathrm{He}}$, the ratio of the processed matter remaining after one cycle of helium shell flash to the ignition mass (Kato \& Hachisu 2004). For low accretion rates $\left(\log \dot{M}_{\mathrm{He}}\left(M_{\odot} \mathrm{yr}^{-1}\right)<-7.6\right)$ a helium detonation occurs which may cause a supernova explosion. In steady state accretion, i.e., in the wind phase or SSS phase, the logarithm of the helium accretion rate is about $-6.0--6.3$ for $\gtrsim 1.3 M_{\odot}$ WDs, and $\eta_{\mathrm{He}}$ is as high as $>0.9$; most of the accreted matter accumulates on the WDs and the rest is lost in the wind. These mass accumulation efficiencies have been incorporated in many binary evolution calculation (see also the discussion after the references).

\section{Acknowledgements}

This research has been supported by the Grant-in-Aid for Scientific Research of the Japan Society for the Promotion of Science (22540254).

\section{References}

Ashok, N. M. \& Banerjee, D. P. K. 2003, AA, 409,1007

Belczynski, K. \& Mikołajewska, J. 1998, MNRAS, 296, 77

Brandi, E., Quiroga, C., Mikołajewska, J., Ferrer, O. E., \& García, L. G. 2009, AA, 497, 815

Cassisi, S., Iben, I. Jr., \& Tornambe, A. 1998, ApJ, 498, 376

Gilfanov, M. \& Bogdán, A. 2010, nature, 463, 924

Goranskij, V., Shugarov, S., Zharova, A., Kroll, P., \& Barsukova, E. A. 2010, Peremennye Zvezdy, 30, 4

Hachisu, I. \& Kato, M. 2003a, ApJ, 590, 445

Hachisu, I. \& Kato, M. 2003b, ApJ, 598, 527

Hachisu, I. \& Kato, M. 2006, ApJS, 167, 59

Hachisu, I. \& Kato, M. 2009a, ApJ, 694, L103

Hachisu, I., Kato, M., Kato, T., \& Matsumoto, K., 2000, ApJ (Letters), 528, L97

Hachisu, I., Kato, M., Kiyota,S., Kubotera, K., Maehara.,H, Nakajima, K., Ishii, Y., Kamada, M., Mizoguchi, S., Nishiyama, S., Sumitomo, N., Tanaka, K., Yamanaka, M., \& Sadakane, K. 2006, ApJ (Letters), 651, L141

Hachisu, I., Kato, M., Kato, T., \& Matsumoto, K. 2000a, ApJ (Letters), 528, L97

Hachisu, I., Kato, M., \& Luna, G. J. M.. 2007, ApJ (Letters), 659, L153

Hachisu, I., Kato, M., \& Nomoto, K. 1996, ApJ (Letters), 470, L97

Hachisu, I., Kato, M., \& Nomoto, K. 1999a, ApJ 522, 487

Hachisu, I., Kato, M., \& Nomoto, K. 2008, ApJ (Letters), 683, L127

Hachisu, I., Kato, M., \& Nomoto, K. 2010, ApJ (Letters), 724, L212

Hachisu, I., Kato, M., Nomoto, K., \& Umeda, H. 1999b, ApJ, 519, 314

Iben, I.,Jr. \& Tutukov, A. V. 1984, ApJ (Suppl.), 54, 335

Kato, M 2011, in: M. Orio \& G.C. Ampama (eds.), proceedings of IAU symo. No 281 (xxxx:xxxx). p. $\mathrm{xx}$

Kato, M. \& Hachisu, I. 1994, ApJ 437, 802

Kato, M. \& Hachisu, I. 2004, ApJ (Letters), 613, L129

Kato, M., Hachisu, I., \& Cassatella, A. 2009, ApJ 704, 1676

Kato, M., Hachisu, I., Kiyota, S., \& Saio, H. 2008, ApJ 684, 1366

Kato, M., Saio, H., \& Hachisu, I. 1989, ApJ 340, 500

Munari, U., Siviero, A., Dallaporta, S., Cherini, G., Valisa, P., \& Tomasella, L. New Astron., 16, 209 
Nomoto, K., Saio, H., Kato, M., \& Hachisu, I. 2007, ApJ 663, 1260

Li, X.-D. \& van den Heuvel, E. P. J. 1997, AA (Letters), 322, L9

van den Heuvel, E. P. J., Bhattacharya, D., Nomoto, K., \& Rappaport, S. A. 1992, AA, 262, 97

\section{Discussion}

WebBink: During He shell flashes, a larger part of the envelope will be lost, which prevent WDs from growing to the Chandrasekhar mass.

KATO: Your argument is based on the paper of Cassisi et al. (1998) which claims that WDs cannot attain the Chandrasekhar mass because most of the envelope is lost during $\mathrm{H} / \mathrm{He}$ shell flashes due to the interaction of the expanded envelope with the companion star. I have counterarguments and comments on the paper as follows.

1. The Cassisi et al. argument is just an assumption. They did not include frictional processes in their calculation, but simply assumed that most of the envelope may be lost once the envelope expands beyond the companion orbit. However, Kato had already shown that frictional processes are ineffective in shell flashes before their work (Kato 1991, ApJ 373, 620; Kato 1991, ApJ 383,761).

2. Nova light curves show no indication of frictional processes. If the companion motion is so effective in mass ejection of the envelope, there should be a clear indication associated with the epoch when the companion emerges from the extended envelope. However, there are no such effects in H/He nova outbursts (Kato \& Hachisu 2011, submitted to ApJ).

3. Cassisi et al. used the old opacity. It is strange that they emphasized that the Los Alamos opacities are very similar to the OPAL opacities, whereas OPAL opacities have a famous large peak at $\log T(\mathrm{~K}) \sim 5.2$, while Los Alamos opacities do not. If one uses the OPAL opacity, the optically thick winds is accelerated on which frictional effects are very small due to small density at the orbit (see Kato \& Hachisu 1994).

4. Cassisi et al. adopted 0.5 and $0.8 M_{\odot}$ WDs and claimed that these are typical initial masses for mass-increasing WDs approaching the Chandrasekhar mass. However, modern SD scenarios (e.g., Hachisu et al., 1999, ApJ, 519, 314; Hachisu et al., 1999, ApJ, 522, 487) show that a possible range of WD masses is higher (namely, 0.9-1.0 $M_{\odot}$ ), and that low mass WDs such as $0.5-0.8 M_{\odot}$ are unlikely to be candidates of SNe Ia.

For these reasons, I disagree with the Cassisi et al. (1998) claim. Nevertheless, the Cassisi et al. arguments have still been cited as a counterargument to the SD scenario by drawing a conclusion that accreting WDs cannot reach the Chandrasekhar mass because of mass ejection due to frictional processes during shell flashes. I found, unfortunately, other presentations of this conference adopt this argument as a counterargument to the $\mathrm{SD}$ scenario, but this is not justified given my reasons above. 